\title{
Digital signal processing in FPGA for particle track reconstruction at the HL-LHC ATLAS
}

\author{
Riccardo Poggi, on behalf of the ATLAS collaboration
}

\begin{abstract}
In the context of the high-luminosity large hadron collider (HL-LHC) upgrade, this work presents the latest update on the design of the FPGA firmware responsible of particle track reconstruction in the pattern recognition mezzanine (PRM) of the hardware-based tracking for the trigger (HTT) system, a subsystem of the ATLAS experiment trigger and data acquisition system. This computationally demanding task relies heavily on two FPGA features: the embedded in silicon digital signal processing (DSP) components and the performance of an available high bandwidth memory (HBM).

The document reports the mathematical algorithm used for track reconstruction and analyses a preliminary performance test. These considerations are then used to provide estimates on the DSP and HBM resource usage in order to prove the feasibility of the firmware design. Finally, key factors for a parallel design are identified and outlook presented.
\end{abstract}

Index Terms-Track reconstruction, DSP, HBM.

\section{INTRODUCTION}

$\mathbf{T}$ HE expected increase in peak luminosity of the highluminosity large hadron collider to $7.5 \times 10^{34} \mathrm{~cm}^{-2} \mathrm{~s}^{-1}$ is driving the ATLAS experiment upgrade strategy for the trigger and data acquisition system [1] towards the implementation of precise hardware-based track reconstruction. The hardware-based tracking for the trigger (HTT) system uses a combination of custom ASICs for pattern recognition and FPGAs to provide the software-based trigger system with access to tracking information, allowing for reduced $p_{\mathrm{T}}$ trigger thresholds for primary lepton selections, while contributing to pile-up mitigation, essential for hadronic signatures. The HTT implementation is based on ATCA carrier boards housing different mezzanine cards. The pattern recognition mezzanine (PRM) is a critical component of this system which mounts the FPGA assigned with the challenging computational task of performing a linear track fit and reconstruction.

In the PRM FPGA firmware architecture (Fig. 1) there are two modules which take care of the aforementioned task: the track fitter block (TFB) and the parameter calculator (PC). To achieve the best possible performance these two entities rely heavily on embedded digital signal processing (DSP) blocks and high bandwidth memory (HBM) [3], both of which can be found in the FPGA model under consideration in this document: the Altera Intel Stratix 10 MX 2100.

Throughout the document rates presented are to be interpreted as data rates and not as clock rates unless otherwise explicitly specified.

Riccardo Poggi, Ph.D. Student at the Département de physique nucléaire et corpusculaire, University of Geneva, Switzerland.

Copyright 2019 CERN for the benefit of the ATLAS Collaboration. CC-BY-4.0 license

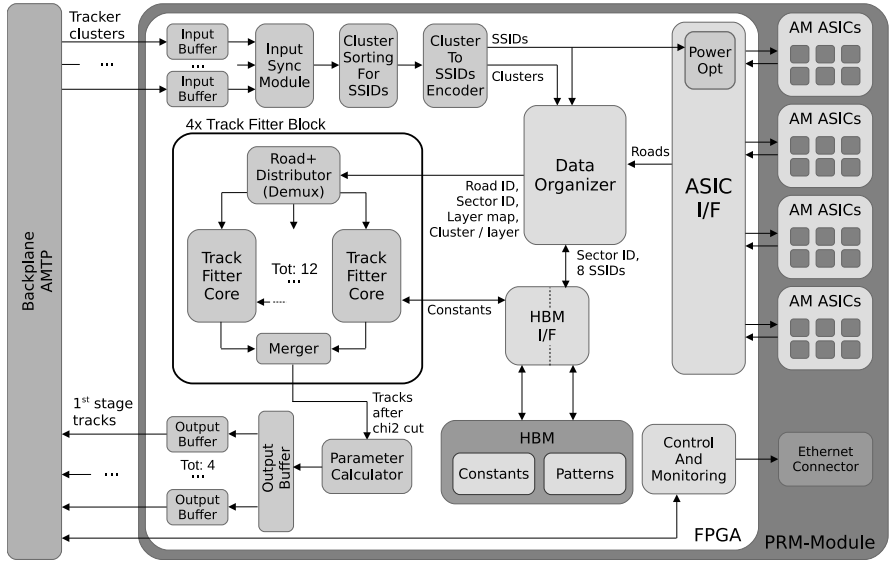

Fig. 1: Data-flow diagram of the major modules of the PRM FPGA. [2]

\section{FUNCTIONAL DESCRIPTION}

In this section the mathematical functions computed by the TFB and PC are described together with a careful breakdown of their arithmetical operations, as introduced in Ref. [4].

\section{A. Track fitter block}

The main function of the TFB is to perform a linear fit, which corresponds to calculating a $\chi^{2}$ value according to the following equation:

$$
\chi^{2}=\sum_{i=1}^{N_{f}}\left[\left(\sum_{j=1}^{N_{\text {coo }}} S_{i j} x_{j}\right)+h_{i}\right]^{2},
$$

where $x_{j}$ are the full-resolution local coordinates of the cluster, while $S_{i j}$ and $h_{i}$ are constant values computed through detector simulation. The constants are unique to a specific physical sector of the ATLAS inner detector and are chosen depending on which sector the clusters belong to.

\begin{tabular}{lll}
\hline \hline Name & Description & \\
\hline$N_{\text {coo }}$ & Number of coordinates & $9 \leq N_{\text {coo }} \leq 12$ \\
$N_{f}$ & $\chi^{2}$ d.o.f & $N_{f}=N_{\text {coo }}-5$ \\
$x_{j}$ & Coordinates & $\operatorname{dim}(x)=N_{\text {coo }}$ \\
$S_{i j}$ & Constants & $\operatorname{dim}(S)=N_{f} \times N_{\text {coo }}$ \\
$h_{j}$ & Constants & $\operatorname{dim}(h)=N_{\text {coo }}-5$ \\
$\chi^{2}$ & Fit result & $\operatorname{dim}\left(\chi^{2}\right)=1$ \\
\hline \hline
\end{tabular}

TABLE I: Description of Eq. 1. 
A breakdown of the type of operations necessary to perform the computation described in Eq. 1 is given in Tab. I and Tab. II.

\begin{tabular}{lll}
\hline \hline & General case & $N_{\text {coo }}=12$ \\
\hline Nr. sums & $\left(N_{\text {coo }}+1\right)\left(N_{\text {coo }}-5\right)-1$ & 90 \\
Nr. mult & $\left(N_{\text {coo }}+1\right)\left(N_{\text {coo }}-5\right)$ & 91 \\
\hline \hline
\end{tabular}

TABLE II: Number of mathematical operations to compute a $\chi^{2}$ as presented in Eq. 1.

1) Missing coordinates: Another activity of the TFB is computing missing coordinates. Due to partial data provided outside the control of the HTT system, it can happen that some components of the input coordinates vector $x_{j}$ could be missing. In these cases an additional set of operations need to be performed before the $\chi^{2}$ computation. The missing coordinates are found through the minimisation of the $\chi^{2}$ presented in Eq. 1, which corresponds to solving the following equation:

$$
\hat{x_{i}}=\sum_{j=1}^{M} C_{i j}^{-1} t_{j}
$$

where $M$ is the number of missing coordinates $\hat{x_{i}}$ to compute, while $C$ and $t$ are given by:

$$
\begin{aligned}
C_{j n} & =\sum_{i=1}^{N_{f}} S_{i \hat{k}_{j}} S_{i \hat{k}_{n}} \\
t_{j} & =-\sum_{i=1}^{N_{f}} S_{i \hat{k}_{j}} h_{i}-\sum_{i=1}^{N_{f}} S_{i \hat{k}_{j}} \sum_{\tilde{k}} S_{i \tilde{k}} x_{\tilde{k}}
\end{aligned}
$$

The $\hat{x}_{i}$ components so computed can then be inserted into the vector $x_{j}$ in place of the missing coordinates.

A breakdown of the type of operations necessary to perform the computation described in Eq. 2 and Eq. 3 is given in Tab. III and Tab. IV.

\begin{tabular}{lll}
\hline \hline Name & Description & \\
\hline$M$ & Nr. of missing coordinates & $1 \leq M \leq 4$ \\
$x_{\tilde{k}}$ & Available coordinates & $\operatorname{dim}(x)=N_{\text {coo }}-M$ \\
$C_{i j}$ & Constants & $\operatorname{dim}(C)=M \times M$ \\
$S h$ & Constants & $\operatorname{dim}(S h)=M$ \\
$S S$ & Constants (sym matrix) & $\operatorname{dim}(S S)=N_{\text {coo }}\left(N_{\text {coo }}+1\right) / 2$ \\
$\hat{x}_{i}$ & Missing coordinates result & $\operatorname{dim}(\hat{x})=M$ \\
\hline \hline
\end{tabular}

TABLE III: Description of Eq. 2 and Eq. 3.

\begin{tabular}{lll}
\hline \hline & General case & $N_{\text {coo }}=12, M=4$ \\
\hline Nr. sums & $M\left(N_{\text {coo }}-M\right)+M(M-1)$ & 44 \\
Nr. mult & $M\left(N_{\text {coo }}-M\right)+M^{2}$ & 48 \\
\hline \hline
\end{tabular}

TABLE IV: Number of mathematical operations to compute $\hat{x_{i}}$ as presented in Eq. 2 and Eq. 3.

\section{B. Parameter calculator}

The PC is the firmware block that comes right after the TFB (see Fig. 1). It performs similar computational operations specified by the following formula:

$$
p_{i}=\sum_{j=1}^{N_{\text {coo }}}\left(B_{i j} x_{j}+q_{i}\right)
$$

where $x_{j}$ are the full-resolution cluster local coordinates, while $B_{i j}$ and $q_{i}$ are constant values unique to the specific sector the coordinates $x_{j}$ belongs to.

A breakdown of the type of operations necessary to perform the computation described in Eq. 4 is given in Tab. V.

\begin{tabular}{lll}
\hline \hline Name & Description & \\
\hline$N_{\text {coo }}$ & Number of coordinates & $9 \leq N_{\text {coo }} \leq 12$ \\
$x_{j}$ & Coordinates & $\operatorname{dim}(x)=N_{\text {coo }}$ \\
$B_{i j}$ & Constants & $\operatorname{dim}(C)=5 \times N_{\text {coo }}$ \\
$q_{i}$ & Constants & $\operatorname{dim}(q)=5$ \\
$p_{i}$ & Track parameters result & $\operatorname{dim}(p)=5$ \\
\hline \hline
\end{tabular}

TABLE V: Detailed description of Eq. 4.

\section{Data-flow}

In the PRM firmware block diagram (Fig. 1) the whole $x_{j}$ vector of cluster coordinates is encoded inside the signal with label "Cluster" which is an output of the data organiser and an input of the TFB. The various TFB and PC constants are represented by the signal with label "Constants", which are fetched from the HBM. A $\chi^{2}$ is computed in the TFB and compared to a threshold. Only coordinates, for which the $\chi^{2}$ satisfies the threshold, are then passed to the PC. The acceptance associated to this requirement is known from simulation to be about $20 \%$ [1].

\section{DigitAl SIgNAL PROCESSING PERFORMANCE TEST}

To support the early design of the PRM an assessment of the performance for the TFB and PC has been done by implementing a matrix multiplication matching the characteristics described in Sec. II, and in particular targeting the worst case scenario of $N_{\text {coo }}=12$ and $M=4$. A sketch of the HDL implementation used is shown in Fig. 2 where the DSP is operated in multiply-accumulate mode [5] taking the input values from local memory. This is coherent with the implementation plan of retrieving the constants from the HBM and keeping them in a local buffer for as long as necessary.

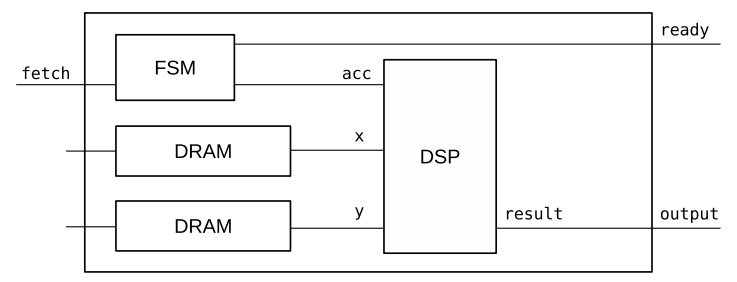

Fig. 2: Schematic view of the firmware module employed for the DSP performance test. Only the important lines, referenced in Fig. 3, are drawn.

The timing diagram shown in Fig. 3 reports the sequence of events and how the block is operated. The fetch signal initiates the retrieval of $x$ and $y$ from the local dual port memories with a latency of two clock cycles. The accumulate acc control 


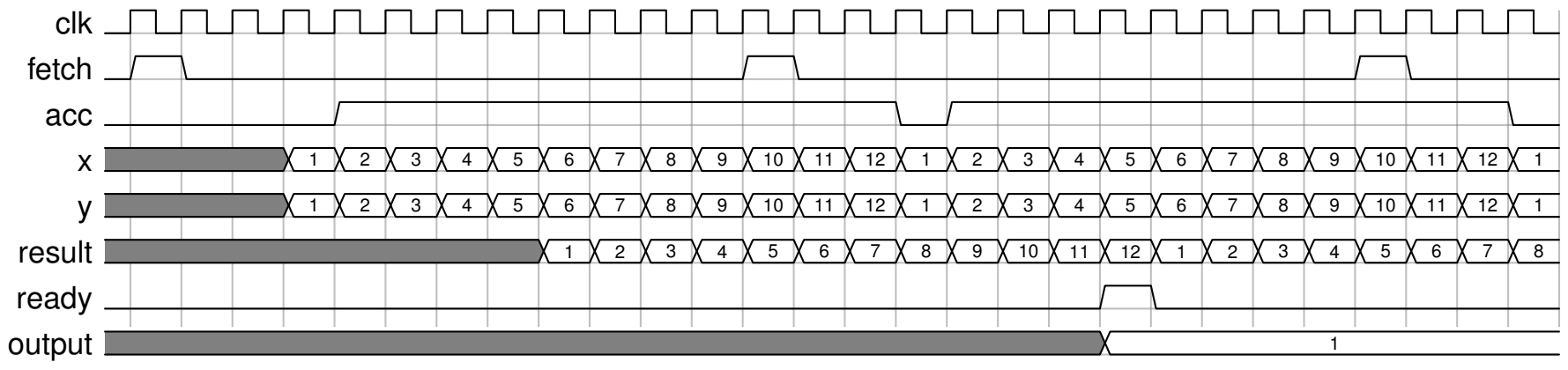

Fig. 3: Timing diagram for the firmware block sketched in Fig. 2 with numeric indices representing the calculation steps.

signal of the DSP is set to "low" for the first input and then set to "high" for the following eleven. After the DSP internal latency of five clock cycles the result register starts holding the partial results: $x_{1} y_{1}, x_{1} y_{1}+x_{2} y_{2}$, etc. until at the twelfth iteration it equals $x_{1} y_{1}+x_{2} y_{2}+\ldots+x_{12} y_{12}$. This last value is then also made available as output, while the second set of input already entered the DSP pipeline without any clock delay, synchronously with the acc control signal set to "low" to restart the accumulation.

There is a trade-off between latency and performance and in this case, since the latency is only at start-up thanks to the pipeline design, the DSP is operated with all internal registers enabled to maximise the performance. With these settings the firmware simulation was able to run with a clock frequency of $500 \mathrm{MHz}$ with the targeted Stratix 10 FPGA device and -2 speed grade. Given the very simplistic implementation of the test, a safe assumption for the estimates in Sec. IV is to consider multiply-accumulate (macc) performances at less than half the rate, i.e. assume $200 \mathrm{M} \frac{\mathrm{macc}}{\mathrm{s} \text { DSP }}$.

\section{RESOURCE ESTIMATES}

A driving factor is the performance target for the PRM of being able to compute four billion $\chi^{2}$ per second (this metric is referred in formulas as $4 \frac{\text { Gfit }}{\mathrm{s}}$ ) [1]. The other values necessary for the evaluation are presented in Sec. II and summarised in Tab. VI and Tab. VII for the same worst case scenario presented in Sec. III ( $N_{\text {coo }}=12$ and $\left.M=4\right)$.

\begin{tabular}{lll}
\hline \hline & Number & Size \\
\hline Macc operations to compute one fit & 140 & \\
\hline Constants used to compute one fit & 140 & $4.5 \mathrm{~kb}$ \\
Constants stored to compute one fit & 317 & $10.1 \mathrm{~kb}$ \\
Total constants stored in HBM & $10 \mathrm{M}$ & $320 \mathrm{Mb}$ \\
\hline \hline
\end{tabular}

TABLE VI: Track fitter block operational summary extracted from Tab. II and Tab. IV assuming single-precision floatingpoint, $N_{\text {coo }}=12$ and $M=4$. The constants are $S, h, S h, S^{2}$ and $C^{-1}$ as defined in Eq. 1, Eq. 2 and Eq. 3.

\section{A. Digital signal processing}

The estimate on the amount of DSP necessary to carry out the mathematical operations described in Sec. II is based on three factors: (1) how many fits ( $\chi^{2}$ from Eq. 1) and track parameters ( $p_{i}$ from Eq. 4 ) the PRM is required to compute, (2)

\begin{tabular}{lll}
\hline \hline & Number & Size \\
\hline Macc operations to compute one set of $p_{i}$ & 60 & \\
\hline Constants used to compute one set of $p_{i}$ & 60 & $1.9 \mathrm{~kb}$ \\
Constants stored to compute one set of $p_{i}$ & 60 & $1.9 \mathrm{~kb}$ \\
Total constants stored in HBM & $1.9 \mathrm{M}$ & $61 \mathrm{Mb}$ \\
\hline \hline
\end{tabular}

TABLE VII: Parameter calculator operational summary extracted from Tab. $\mathrm{V}$ assuming single-precision floating-point and $N_{\text {coo }}=12$. Constants: $B_{i j}, q_{i}$ as defined in Eq. 4.

which and how many mathematical operations are necessary to compute these quantities, (3) how many of these operations can a single DSP carry out in a given time.

1) Track fitter block: The following formula estimates how many DSP would be necessary to instantiate for the TFB:

$$
140 \frac{\text { macc }}{\text { fit }} \times \frac{4 \frac{\text { Gfit }}{\mathrm{s}}}{200 \mathrm{M} \frac{\mathrm{macc}}{\mathrm{s} \text { DSP }}}=2800 \mathrm{DSP}
$$

where the value of $140 \frac{\mathrm{macc}}{\mathrm{fit}}$ is taken from Tab. VI.

2) Parameter calculator: After the $\chi^{2}$ computation from the TFB a decision is made to discard all tracks with a $\chi^{2}$ value below a certain threshold. The acceptance corresponding to this threshold is known from simulation to correspond to about $20 \%$ [1]. As a consequence, considering from Tab. VII that the number of multiply-accmulate operations for one parameter calculation (pc) is about $60 \frac{\text { macc }}{\mathrm{pc}}$ we get the following:

$$
60 \frac{\mathrm{macc}}{\mathrm{pc}} \times \frac{(4 \times 0.2) \frac{\mathrm{Gpc}}{\mathrm{s}}}{200 \mathrm{M} \frac{\mathrm{macc}}{\mathrm{s} \mathrm{DSP}}}=240 \mathrm{DSP}
$$

\section{B. High bandwidth memory}

The HBM stores the various constants necessary to compute the $\chi^{2}$ and the track parameters (Fig. 1). These values are retrieved from the HBM and kept in a local buffer close to the computing core. The necessary bandwidth is therefore estimated by multiplying the rate at which the HBM is accessed with the size of the payload requested.

1) Track fitter block: Since on average the same set of track fitter constants can be used ten times in a row [1], we get the following:

$$
\mathrm{BW} \approx 4 \frac{\mathrm{Gfit}}{\mathrm{s}} \times \frac{560 \mathrm{~B}}{10 \mathrm{fit}}=224 \frac{\mathrm{GB}}{\mathrm{s}}
$$

where $4 \frac{\text { Gfit }}{\mathrm{s}}$ is the performance requirement of the PRM, and $560 \mathrm{~B}$ is the payload corresponding to the retrieval of one set 
of 140 constants in single-precision floating-point as expressed in Tab. VI.

2) Parameter calculator: In a similar way for the parameter calculator we get:

$$
\mathrm{BW} \approx 0.2 \frac{\mathrm{pc}}{\mathrm{fit}} \times 4 \frac{\mathrm{Gfit}}{\mathrm{s}} \times 240 \frac{\mathrm{B}}{\mathrm{pc}}=192 \frac{\mathrm{GB}}{\mathrm{s}}
$$

where $0.2 \frac{\mathrm{pc}}{\mathrm{fit}}$ is the ratio between the number of track parameter calculations and track fittings performed in the PRM, $4 \frac{\text { Gfit }}{\mathrm{s}}$ is the required performance of the PRM, and $240 \frac{\mathrm{B}}{\mathrm{pc}}$ is the payload corresponding to retrieving the necessary 60 constants as expressed in Tab. VII.

\section{PARALlel Design}

Fig. 1 shows the plan for having multiple track fitter cores (TFCs) operating in parallel inside the TFB and Fig. 5 shows a parallel paradigm which can be used for the implementation of the TFC. The feasibility of this design is contingent on the capability of the HBM to provide the inputs with enough speed and parallelisation. Since there is a trade-off between resource usage and performance, another factor to take into account regards the DSP. To guide the development of the TFC, Fig. 6 shows the inverse relationship present in a single TFC between latency and DSP resource allocation necessary to achieve the PRM target throughput for different clock frequencies.

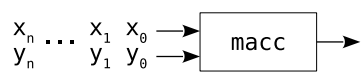

Fig. 4: Data-flow representation of one multiply-accumulate processing block which sequentially processes the input data.

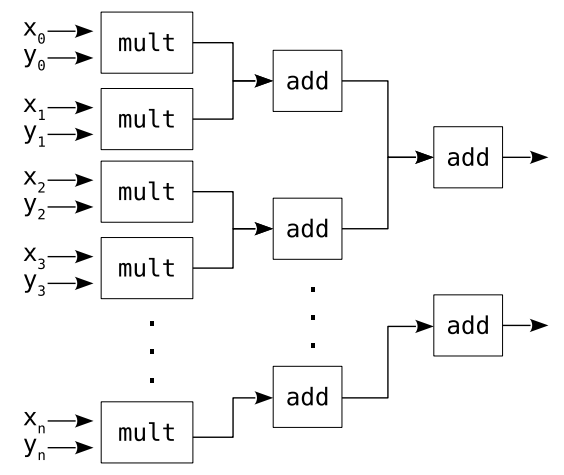

Fig. 5: Data-flow representation of a hierarchical structure with all multiplications done in parallel at the beginning.

\section{CONCLUSION}

The DSP and HBM resource usages derived in Sec. IV show for the DSP an estimated total occupancy of about $76 \%$ w.r.t the DSP resources available in the targeted FPGA model, while for HBM there is a negligible memory occupancy and a bandwidth utilisation at about $80 \%$ of the nominal value declared by the FPGA vendor.

For the future we plan to continue the development and to provide performance simulation for a first TFC prototype.

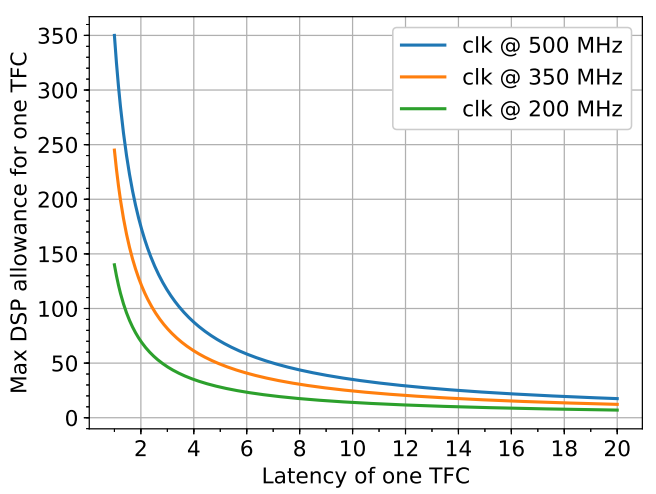

Fig. 6: Maximum DSP allowance for one single TFC as a function of its latency (number of clock cycles between two $\chi^{2}$ values), given a total allowance of 2800 DSP for the TFB and the required PRM data throughput of $4 \frac{\text { Gfit }}{\mathrm{s}}$.

\begin{tabular}{lllllll}
\hline \hline Block & \multicolumn{2}{c}{ DSPs } & \multicolumn{2}{c}{ HBM usage } & \multicolumn{2}{c}{ HBM BW } \\
\hline TFB & 2800 & $71 \%$ & $40 \mathrm{MB}$ & $0.7 \%$ & $224 \mathrm{~GB} / \mathrm{s}$ & $44 \%$ \\
PC & 240 & $5 \%$ & $7.6 \mathrm{MB}$ & $0.09 \%$ & $192 \mathrm{~GB} / \mathrm{s}$ & $37 \%$ \\
\hline Tot & 3040 & $76 \%$ & $48 \mathrm{MB}$ & $0.8 \%$ & $416 \mathrm{~GB} / \mathrm{s}$ & $81 \%$ \\
\hline \hline
\end{tabular}

TABLE VIII: Resource usage summary. Percentages w.r.t. nominal values for Intel Altera Stratix 10 MX 2100 [6].

Regarding the HBM, since the bandwidth plays such a critical role in the feasibility of a parallel computational implementation, further studies will be performed to better understand the HBM access timings from the TFB and PC. As a first step, a test on the HBM similar to the one presented for the DSP would provide the necessary information to derive the bandwidth efficiency, a factor which still needs to be included into the HBM utilisation estimates.

\section{REFERENCES}

[1] ATLAS Collaboration, "Technical Design Report for the Phase-II Upgrade of the ATLAS TDAQ System," CERN, Geneva, Tech. Rep. CERN-LHCC-2017-020. ATLAS-TDR-029, Sep 2017. [Online]. Available: http://cds.cern.ch/record/2285584

[2] R. Poggi, "Design of the ATLAS phase-II hardware-based tracking processor," Nuclear Instruments and Methods in Physics Research Section A: Accelerators, Spectrometers, Detectors and Associated Equipment, 2018. [Online]. Available: http://www.sciencedirect.com/ science/article/pii/S0168900218316309

[3] M. Deo, J. Schulz, and L. Brown. (2017) Intel stratix $10 \mathrm{mx}$ devices solve the memory bandwidth challenge. http://my.url.com/. [Online]. Available: https://www. intel.com/content/dam/www/programmable/us/en/pdfs/literature/wp/ wp-01264-stratix 10mx-devices-solve-memory-bandwidth-challenge.pdf

[4] A. Annovi, F. Crescioli, M. Dell'Orso, P. Giannetti, G. Punzi, and G. Volpi, "Linear Fit within Missing-Hit Roads in FTKSim," CERN, Geneva, Tech. Rep. ATL-COM-DAQ-2017-161, Mar 2016. [Online]. Available: https://cds.cern.ch/record/2290696

[5] Intel. (2018) Intel stratix 10 variable precision dsp blocks user guide. [Online]. Available: https://www.intel.com/content/dam/www/ programmable/us/en/pdfs/literature/hb/stratix-10/ug-s10-dsp.pdf

[6] - (2018) Intel stratix 10. [Online]. Available: https://www.intel.com/content/dam/www/programmable/us/en/pdfs/ literature/pt/stratix-10-mx-product-table.pdf 